\title{
The Bijectivity of the Tight Frame Operators in Lebesgue Spaces
}

\author{
Kai-Cheng Wang, Chi-I. Yang, and Kuei-Fang Chang
}

\begin{abstract}
The motivation of this dissertation mainly is which affine frame wavelet systems span Lebesgue spaces have been investigated less. The technique we used is analogous to technique of Calderón-Zygmund operators, but we rely on Calderón-Zygmund decomposition theorem. We prove our main results without smoothness assumption on frame wavelets. We prove that affine tight frame wavelets span Lebesgue spaces under the condition, we also show that the affine tight frame operator extends from $L^{2}(\mathbb{R})$ to a bounded, linear and bijective

operator on $L^{p}(\mathbb{R})$, for $1<p<\infty$. Under such condition, the affine orthonormal basis of $L^{2}(\mathbb{R})$ is also an unconditional basis
\end{abstract} for $L^{p}(\mathbb{R}), 1<p<\infty$.

Index Terms-Bijective, frames, orthogonal basis, unconditional basis, wavelets.

\section{INTRODUCTION}

The conditions of many problems force the use of a set of basis $\left\{\psi_{i}\right\}_{i=1}^{\infty}$ of a Hilbert space $H$ which can not be orthonormal (often because of uncertainty principles). Lastly, if we can disregard even linear independence, we end up with the "frames" whose theory was first developed by Duffin and Schaeffer [1]. A frame $\left\{\psi_{i}\right\}_{i=1}^{\infty}$ acts like an overcomplete set in linear algebra where one can express any $f \in H$ with but not necessarily in a unique way since the elements $\psi_{i}$ need not to be linearly independent. In this paper, we focus on tight frames which their canonical dual frame has the same structure as the frames themselves. This implies many advantages. Indeed, it is not only difficult to control the behavior of the dual frames, but also nothing can guarantee the decay of the dual frames. Tight frames act like orthogonal bases but are without linear independence. Historically, [2], [3], [4] use the Calderón-Zygmund operators; [5], [6] use multiresolution analysis and Marcinkiewicz interpolation theorem. We prove that the frame operator associated the affine tight frame with the condition extends from $L^{2}(\mathbb{R})$ to a bounded, linear and bijective operator on $L^{p}(\mathbb{R})$, for $1<p<\infty$. By the way, we can use the same technique to the case for an orthonormal wavelet case. We list our main

Manuscript received November 8, 2013; revised January 14, 2014

Kai-Cheng Wang is with Ph.D. Program in Mechanical and Aeronautical Engineering, Feng-Chia University, 40724 Tai-Chung, Taiwan (e-mail: gtotony98@gmail.com).

Chi-I. Yang and Kuei-Fang Chang are with Department of Applied Mathematics, Feng-Chia University, 40724 Tai-Chung, Taiwan (e-mail: yesheslhamo@gmail.com, kfchang@math.fcu.edu.tw). results.

Theorem 1.1: The frame operator $S$ associated $\mathcal{F}_{\psi}$ (define later) is bijective on $L^{p}(\mathbb{R}), 1<p<\infty$ and hence $\mathcal{F}_{\psi}$ is dense in the Lebesgue spaces. Moreover,

$$
C\|f\|_{p} \leq\|S f\|_{p} \leq D\|f\|_{p},
$$

for all $f \in L^{p}(\mathbb{R}), 1<p<\infty$ and some constants $0<C, D<\infty$.

Theorem 1.2: Let $\mathcal{F}_{\psi}$ be an orthonormal basis of $L^{2}(\mathbb{R})$. Then,

1) $T$ is of type weak $(1,1)$ and of type $(p, p)$, for all $1<p<\infty$. Moreover,

$$
\frac{1}{C}\|f\|_{p} \leq\|T f\|_{p} \leq C\|f\|_{p}
$$

where $C$ does not depend on $f$, for all $f \in L^{p}(\mathbb{R}), 1<p<\infty$.

2) $\mathcal{F}_{\psi}$ forms an unconditional basis for $L^{p}(\mathbb{R})$, for al $l$ $1<p<\infty$.

3) If $1<p \leq 2, \mathcal{F}_{\psi}$ is Besselian for $L^{p}(\mathbb{R})$ and there exists $c_{p}, C_{p}>0$ such that

$$
c_{p}\left\|\left\{a_{j, k}\right\}\right\|_{2} \leq\left\|\sum_{j, k \in \mathbb{Z}} a_{j, k} \psi_{j, k}\right\|_{p} \leq C_{p}\left\|\left\{a_{j, k}\right\}\right\|_{p},
$$

for all scalars $a_{j, k}$.

4) If $2 \leq p<\infty, \mathcal{F}_{\psi}$ is Hilbertian for $L^{p}(\mathbb{R})$ and there exists $c_{p}, C_{p}>0$ such that

$$
c_{p} \cdot\left\|\left\{a_{j, k}\right\}\right\|_{p} \leq\left\|\sum_{j, k \in \mathbb{Z}} a_{j, k} \psi_{j, k}\right\|_{p} \leq C_{p}\left\|\left\{a_{j, k}\right\}\right\|_{2},
$$

for all scalars $a_{j, k}$.

5) $\mathcal{F}_{\psi}$ is simultaneously Besselian and Hilbertian if and only if $p=2$

\section{PRELIMINARIES AND Notations}

Bases in this paper are Schauder bases. In [7], it mentions a special class which satisfies Wiener condition. Let $\psi$ be a measurable function on $\mathbb{R}$ and satisfies the following inequality: 


$$
\|\psi\|_{W\left(L^{\infty}, l^{1}\right)}:=\sum_{k \in \mathbb{Z}[0,1)} \sup _{[}|\psi(x+k)|<\infty
$$

where $\chi$ is the characteristic function. We consider that

$$
\begin{aligned}
& \int_{\mathbb{R}}|\psi(x)|^{p} d x=\int_{0}^{1}\left(\sum_{k \in \mathbb{Z}} \psi(x+k) \mid\right)^{p} d x \\
& \leq\left(\sum_{k \in \mathbb{Z}[0,1)} \sup |\psi(x+k)|\right)^{p}=\|\psi\|_{W\left(L^{\infty}, l^{1}\right)}^{p} .
\end{aligned}
$$

It follows that if (1) holds, we have $\psi \in L^{p}(\mathbb{R})$, for all $1 \leq p \leq \infty$. Let $T$ be a mapping from $L^{p}(\mathbb{R})$ to $L^{q}(\mathbb{R})$, for all $1 \leqslant p, \quad q \leqslant \infty$. Then, $T$ is of type $(p, q)$ if

$\|T(f)\|_{q} \leq A\|f\|_{p}, f \in L^{p}(\mathbb{R})$ where $A$ does not depend on $f$. Similarly, $T$ is of weak type $(p, q)$ if

$$
m\{x:|T f(x)|>\alpha\} \leq\left(\frac{A\|f\|_{p}}{\alpha}\right)^{q}, q<\infty, \alpha>0
$$

where $A$ does not depend on $f$ or $\alpha$, and $m$ is the Lebesgue measure. We note that an operator of type $(p, q)$ is of weak type $(p, q)$. A sequence $\left\{\psi_{i}\right\}_{i=1}^{\infty}$ of elements $H$ is a frame for $H$ if there exists constants $A, B>0$ such that

$$
A\|f\|^{2} \leq \sum_{i=1}^{\infty}\left|<f, \psi_{i}>\right|^{2} \leq B\|f\|^{2},
$$

for all $f \in H$. Let $\left\{\psi_{i}\right\}_{i=1}^{\infty}$ be a frame with frame operator $S$ (see [8]). Then,

$$
f=\sum_{i=1}^{\infty}<f, S^{-1} \psi_{i}>\psi_{i},
$$

for all $f \in H$. The series converges unconditionally in $H$. $\left\{S^{-1} \psi_{i}\right\}_{i=1}^{\infty}$ is called the dual of $\left\{\psi_{i}\right\}_{i=1}^{\infty}$. We also list some properties which are needed to our results.

1) Let $H=L^{2}(\mathbb{R})$. We denote the frame operator $S$ of $\left\{\psi_{i}\right\}_{i=1}^{\infty}$ as $S f:=\sum_{i=1}^{\infty}<f, \psi_{i}>\psi_{i}$, for all $f \in L^{2}(\mathbb{R}) . T$ is called the pre-frame operator or the synthesis operator which is bounded.

$T: l^{2}(\mathbb{N}) \rightarrow H, T\left\{c_{i}\right\}_{i=1}^{\infty}:=\sum_{i=1}^{\infty} c_{i} \psi_{i}, S=T T^{*}=T^{*} T$.

2) Both $S$ and $S^{-1}$ are of type $(2,2)$, bounded, invertible, self-adjoint and positive [8].

3) A frame may have several duals; a dual which is not the canonical dual is called an alternate dual. $\left\{S^{-1} \psi_{i}\right\}$ may not have wavelet structure.

4) $\left\{S^{-1} \psi_{i}\right\}$ is also a frame in $H$ and its frame operator is $S^{-1}$

5) For a tight frame $\left\{\psi_{i}\right\}_{i=1}^{\infty},\left\{S^{-1} \psi_{i}\right\}=\left\{\frac{1}{A} \psi_{i}\right\}$, and $A$ is the tight frame bound.
There are rich information about frames can be found in [2].

\section{PROOFS}

We start at the $L^{p}$ - boundedness of frame operators. Let $\left\{\psi_{j, k}: j, k \in \mathbb{Z}\right\} \quad$ be a frame in $L^{2}(\mathbb{R})$ where $\psi_{j, k}:=2^{j / 2} \psi\left(2^{j} \cdot-k\right)$. We say $\psi \in \mathcal{M}$ if $\psi$ satisfies

$\int_{0}^{\infty} \log _{2}(1+x) \sup _{x \leq|y|}|\psi|(y) d x<\infty$. We list two things that need to be noticed.

1) If $\psi \in \mathcal{M}, \psi$ is also belong to $W\left(L^{\infty}, l^{1}\right)$ so that $\psi$ is belong to $L^{p}(\mathbb{R}), 1 \leq p \leq \infty$.

2) Our result can be applied to all compactly support orthonormal/tight frame wavelets.

For all $f \in L^{1}(\mathbb{R})$, by Calderón-Zygmund decomposition theorem [9], there exists a collection $\Omega \subset \mathbb{Z}^{2}$ such that intervals $\quad\left\{I_{m, n}\right\}_{(m, n) \in \Omega} \quad$ are disjoint, $I_{m, n}:=\left[2^{-m} n, 2^{-m}(n+1)\right),|f(x)| \leq \alpha$, almost everywhere on $F:=\mathbb{R} \backslash \bigcup_{(m, n) \in \Omega} I_{m, n}$, for all $\alpha>0$, and also for all $(m, n) \in \Omega$,

$$
\begin{array}{r}
\alpha<2^{m} \int_{I_{m, n}}|f| \leq 2 \alpha \text {. So we have } \\
\sum_{(m, n) \in \Omega} \alpha 2^{-m}<\|f\|_{1} .
\end{array}
$$

We denote an affine tight frame $\mathcal{F}_{\psi}:=\left\{\psi_{j, k}: \psi \in \mathcal{M}, j, k \in \mathbb{Z}\right\}$, and $P_{m}$ is the projection from $L^{2}(\mathbb{R})$ onto $\mathcal{V}_{m}:=\overline{\operatorname{span}}\left\{\psi_{j, k}: j, k \in \mathbb{Z}, j<m\right\}$, and let $\left\{S^{-1} \psi_{j, k}: j, k \in \mathbb{Z}\right\}$ be the canonical dual frame of $\mathcal{F}_{\psi}$,

$$
\begin{aligned}
& P_{m}(f):=\sum_{\substack{j, k \in \mathbb{Z} \\
j<m}}<f, S^{-1} \psi_{j, k}>\psi_{j, k} \\
& =\frac{1}{A} \sum_{\substack{j, k \in \mathbb{Z} \\
j<m}}<f, \psi_{j, k}>\psi_{j, k},
\end{aligned}
$$

for all $f \in L^{2}(\mathbb{R})$. For all $f \in L^{1} \cap L^{2}(\mathbb{R})$, we set

$$
\begin{aligned}
g & :=f \chi_{F}+\sum_{(m, n) \in \Omega} P_{m}\left(f \chi_{I_{m, n}}\right), \\
h & :=f-g=\sum_{(m, n) \in \Omega}\left[f \chi_{I_{m, n}}-P_{m}\left(f \chi_{I_{m, n}}\right)\right] .
\end{aligned}
$$

It follows that $S h$

$$
=\sum_{(m, n) \in \Omega} \sum_{\substack{j, k \in \mathbb{Z} \\ j^{\prime}, k^{\prime} \in \mathbb{Z} \\ j^{\prime} \geq m}}<f \chi_{I_{m, n}}, \psi_{j^{\prime}, k^{\prime}}><\psi_{j, k}, \psi_{j^{\prime}, k^{\prime}}>\psi_{j, k} .
$$

Claims that $S$ is of weak type $(1,1)$ and of type $(p, p), 1<p<\infty$ which relies on the same idea of [5], [6], but we don't have complicated estimations. 
Theorem 3.1: Under notations as above, then, $S$ is weak type $(1,1)$ and of type $(p, p)$, for all $1<p<\infty$.

Finally, we prove Theorem 1.2. by defining an operator on $L^{p}(\mathbb{R}) \quad$ as $\quad T f:=\sum_{j, k \in \mathbb{Z}} \epsilon_{j, k}<f, \psi_{j, k}>\psi_{j, k}, \quad$ for $\quad$ all $\epsilon_{j, k}= \pm 1, f \in L^{p}(\mathbb{R})$ since the feature of $T$ is similar to the frame operator $S$ So, we can conclude that $T$ is of type ( $p$, $p$ ), for all $1<p<\infty$.

Proof of Theorem 3.1: First, we claim that, for all $\alpha>0$,

$\|g\|_{2}^{2} \leq \alpha A_{1}\|f\|_{1}$ where constant $A_{1}$ does not depend on $f$ and $\alpha$. Assuming, by Dirichlet test,

$$
\begin{aligned}
& M_{1}:=A^{-2}\|\psi\|_{2}^{2} \sum_{\substack{j, k \in \mathbb{Z} \\
j<m<m^{\prime}}} 2^{\frac{j-m}{2}} \sup _{\substack{I_{m, n} \\
j<m}}|\psi|\left(2^{j} x+k\right)<\infty \text {, } \\
& \left\|\sum_{(m, n) \in \Omega} P_{m}\left(f \chi_{I_{m, n}}\right)\right\|_{2}^{2} \\
& =\int_{\mathbb{R}} \sum_{(m, n) \in \Omega\left(m^{\prime}, n^{\prime}\right) \in \Omega} P_{m}\left(f \chi_{I_{m, n}}\right) \overline{P_{m}\left(f \chi_{I_{m, n}, n}\right)} \\
& \leq 4 \alpha M_{1} \sum_{(m, n) \in \Omega} \int_{I_{m, n}}|f| \\
& \left(\sum_{\substack{\left(m, n^{\prime}\right) \in \Omega \\
m \leq m^{\prime}}} \sum_{\substack{j, k^{\prime} \in \mathbb{Z} \\
j<m<m^{\prime}}} 2^{\frac{j^{\prime}-m^{\prime}}{2}} \sup _{\substack{I_{m^{\prime}, n^{\prime}} \\
j<m^{\prime}}}|\psi|\left(2^{j^{\prime}} x+k^{\prime}\right)\right) \\
& \leq 4 \alpha M_{1} \sum_{(m, n) \in \Omega} \int_{I_{m, n}}|f| \\
& \left(\sum_{p \in \mathbb{Z}} \sum_{m^{\prime}=m}^{\infty} \sum_{\substack{n^{\prime}=p^{\frac{m^{\prime}-m}{2}} \\
(p+1) 2^{\frac{m^{\prime}-m}{2}}-1}} \sum_{\substack{j, k \in \mathbb{Z} \\
j^{\prime}<m<m^{\prime}}} 2^{\frac{j^{\prime}-m^{\prime}}{2}} \sup _{2^{\frac{m}{2}} I_{m^{\prime}, n^{\prime}}}|\psi|\left(2^{j^{\prime}-\frac{m^{\prime}}{2}} x+k^{\prime}\right)\right) \\
& \leq 4 \alpha M_{1} \sum_{(m, n) \in \Omega} \int_{I_{m, n}}|f| \\
& \left(\sum_{p \in \mathbb{Z}} \sum_{\substack{j, k^{\prime} \in \mathbb{Z} \\
j<m<m^{\prime}}} 2^{\frac{j^{\prime}-m}{2}} \sup _{2^{j-\frac{m}{2}} I_{p}}|\psi|\left(x+k^{\prime}\right)\right) \leq 16 \alpha M_{1} N\|f\|_{1}
\end{aligned}
$$

where, by Dirichlet test, $N:=\sum_{p \in \mathbb{Z}} \sum_{\substack{j, k^{\prime} \in \mathbb{Z} \\ j<m<m^{\prime}}} 2^{\frac{j-m}{2}} \sup _{2^{j-\frac{m}{2}} I_{p}}|\psi|\left(x+k^{\prime}\right)<\infty . \quad$ Indeed, it follows from if $m \leq m^{\prime}$ and $p 2^{\frac{m^{\prime}-m}{2}} \leq n^{\prime}<(p+1) 2^{\frac{m^{\prime}-m}{2}}$, then $2^{\frac{-m^{\prime}}{2}} I_{m^{\prime}, n^{\prime}} \subset I_{p}$. We also have, under such $n^{\prime}$, $\left|\bigcup_{2}^{\frac{-m^{\prime}}{2}} I_{m, n}\right|<\left|I_{p}\right|=2^{\frac{-m}{2}}$. It leads that, for each $p$, we have

$$
\sum_{m^{\prime}=m}^{\infty} \sum_{n^{\prime}=2^{\frac{m^{\prime}-m}{2}}}^{(p+1) 2^{\frac{m^{\prime}-m}{2}}-1} 2^{\frac{-m i}{2}}<4 \cdot 2^{\frac{-m}{2}} \text {. When we take any finite }
$$

terms of $p, j$ and $k$,

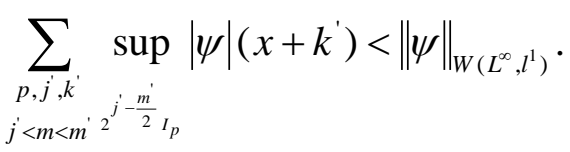

Next, $\quad \int_{\mathbb{R}}\left|f \chi_{F}\right|^{2} \leq \alpha \int_{F}|f| \leq \alpha\|f\|_{1}, \quad$ it $\quad$ leads to $\|g\|_{2}^{2} \leq\left(16 M_{1} N+1\right) \alpha\|f\|_{1}$. Secondly, we set

$$
\begin{gathered}
I_{m, n}^{*}:=\left[2^{-m}(n-1), 2^{-m}(n+2)\right), F^{*}:=\mathbb{R} \backslash \bigcup_{(m, n) \in \Omega} I_{m, n}^{*}, \\
\varphi(x):=\sup _{x \leq|y|}|\psi|(y), x \geq 0 . \\
\int_{F^{*}}|S h| \leq \int_{\mathbb{R} \backslash I_{m, n}^{*}}|S h| \\
\leq\|\psi\|_{1}\left(\sum_{(m, n) \in \Omega} \int_{I_{m, n}}|f|\right)\left(\sum_{j, k^{\prime} \in \mathbb{Z} 2^{j} I_{m, n}+k^{\prime}} \sup _{j \geq m}|\psi|(x)\right) \\
\left(\sum_{j, k \in \mathbb{Z}} \sup _{x \in \mathbb{R}}|\psi|\left(2^{j} x+k\right)\right) \int_{\mathbb{R} \backslash I_{m, n}^{*}} 2^{j / 2}\left|\psi \psi_{j, k}\right| \\
\leq B\|f\|_{1 .} .
\end{gathered}
$$

Indeed, fixing $m, n$, we consider

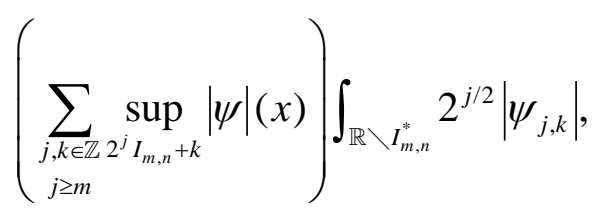

and breaking $k$ into parts,

$$
\begin{aligned}
& \sum_{j=m}^{\infty}\left(\sum_{k \leq-2^{j-m+1}}+\sum_{k>2^{j-m-1}}\right) \sup _{\left[k, k+2^{j-m}\right)}|\psi|(x) \\
& \left(\int_{-\infty}^{k-2^{j-m}}+\int_{2^{j-m+1}+k}^{\infty}\right)|\psi|
\end{aligned}
$$

$$
\begin{aligned}
& \leq 2\|\psi\|_{1} \sum_{j=m}^{\infty}\left(\sum_{-\infty}^{k=-2 \cdot j^{j-m}} \varphi\left(k+2^{j-m}\right)\right. \\
& \left.+\sum_{k=\left[\frac{1}{2} 2^{j-m}\right\rceil}^{\infty} \varphi(k)\right)
\end{aligned}
$$

$$
\leq 4\|\psi\|_{1} \sum_{j=m}^{\infty} \sum_{k=\left\{\frac{1}{2} 2^{j-m}\right\rceil}^{\infty} \varphi(k)
$$




$$
\begin{aligned}
& \leq 4\|\psi\|_{1}\left(\varphi(0)+\sum_{p=1}^{\infty}\left(\left\lfloor\log _{2} p\right\rfloor+2\right) \varphi(p)\right) \\
& \leq 4\|\psi\|_{1}\left(\varphi(0)+\int_{1}^{\infty}\left(\log _{2} x+2\right) \varphi(x) d x\right) . \\
& \sum_{j=m-2^{j-m+1}<k \leq 2^{j-m-1}}^{\infty} \sup _{\left[k, k+2^{j-m}\right)}|\psi|(x)\left(\int_{-\infty}^{k-2^{j-m}}+\right. \\
& \left.\int_{2^{j-m+1}+k}^{\infty}\right)|\psi| \\
& \leq 2\|\psi\|_{1} \sum_{j=m}^{\infty} \sum_{2^{j-m+1}<k \leq 2^{j-m-1}\left[k, k+2^{j-m}\right)}|\psi|(x) \\
& \leq 4\|\psi\|_{1} \sum_{j=m}^{\infty} \sum_{p=0}^{2^{j-m+1}} \varphi(p) \\
& \leq 4\|\psi\|_{1}\left(\varphi(0)+\int_{1}^{\infty}\left(\log _{2} x+2\right) \varphi(x) d x\right) .
\end{aligned}
$$

This argument also leads to

$$
\begin{aligned}
& \sum_{\substack{j, k \in \mathbb{Z} 2^{j} I_{m, n}+k \\
j \geq m}} \sup _{I^{\prime}}|\psi|(x)<\infty, \sum_{j, k \in \mathbb{Z} 2^{j} I_{m, n}+k} \sup _{I^{\prime}}|\psi|(x) \\
& \leq\|\psi\|_{W\left(L^{\infty}, l^{1}\right)}+\sum_{\substack{j, k \in \mathbb{Z} 2^{j} I_{m, n}+k \\
j \geq m}} \sup _{I_{m, n}}|\psi|(x)<\infty .
\end{aligned}
$$

The summation $\sum_{j, k \in \mathbb{Z}} \sup _{x \in \mathbb{R}}|\psi|\left(2^{j} x+k\right)$ also finite which it is not only independent with $x$, but there exists a constant $D, \sum_{j, k \in \mathbb{Z}} \sup _{x \in \mathbb{R}}|\psi|\left(2^{j} x+k\right)=D \sum_{j, k \in \mathbb{Z} 2^{j} I_{m, n}+k}|\psi|(x)$.

Finally, for all $f \in L^{1} \cap L^{2}(\mathbb{R}), \alpha>0$, we note that $m\left\{\mathbb{R} \backslash F^{*}\right\}<3 / \alpha\|f\|_{1}$ (from (2)) and $S$ is of type $(2,2)$.

$$
\begin{aligned}
& m\{x:|S f|>\alpha\} \\
& \leq m\{x:|S g|>\alpha / 2\}+m\{x:|S h|>\alpha / 2\} \\
& \leq m\left\{x:|S g|^{2}>\alpha^{2} / 4\right\}+(2 B+3) / \alpha\|f\|_{1} \\
& \leq\left[\left(4 A_{1}+2 B+3\right) / \alpha\right]\|f\|_{1} .
\end{aligned}
$$

So, $S$ is of weak type $(1,1)$. By Marcinkiewicz interpolation theorem, it leads to that $S$ is of type $(p, p)$ hence $S$ is of weak type $(p, p)$, for all $1<p<2$ and thus by duality for all $p, 1<p<\infty$.

Proof of Theorem 1.1: From Theorem 3.1., for all $f \in L^{2} \cap L^{p}(\mathbb{R})$, we have

$$
\begin{gathered}
\|f\|_{p}=\sup _{\|h\|_{q}=1, h \in L^{2}(\mathbb{R})}\left|\int_{\mathbb{R}} f h\right| \\
\leq \sup _{\|h\|_{q}=1, h \in L^{2}(\mathbb{R})}\left|<S f, S^{-1} h>_{L^{2}(\mathbb{R})}\right| \leq \frac{1}{C}\|S f\|_{p},
\end{gathered}
$$

for some constant $\frac{1}{C}>0$ and $1 / p+1 / q=1$. Since
$L^{2} \cap L^{p}(\mathbb{R})$ is dense in $L^{p}(\mathbb{R})$, we take $\left\{f_{k}\right\} \subset L^{2} \cap L^{p}(\mathbb{R})$ such that $f_{k}$ converges to $f$ in $L^{p}-$ norm, for all $f \in L^{p}(\mathbb{R}), 1<p<\infty$,

$$
\|f\|_{p}=\lim _{k \rightarrow \infty}\left\|f_{k}\right\|_{p} \leq \frac{1}{C} \lim _{k \rightarrow \infty}\left\|S f_{k}\right\|_{p}=\frac{1}{C}\|S f\|_{p} .
$$

We denote $S^{*}$ is the adjoint of $S$ which is on $L^{q}(\mathbb{R})$ into $L^{q}(\mathbb{R})$. Following (3),

$$
\begin{aligned}
& \|f\|_{p} \cdot 1 \leq \frac{1}{C}\|S f\|_{p}=\frac{1}{C} \sup _{\|g\|_{q}=1}\left|\int_{\mathbb{R}}(S f) g\right| \\
& =\frac{1}{C} \sup _{\|g\|_{q}=1}\left|\int_{\mathbb{R}} f\left(S^{*} g\right)\right| \leq \frac{1}{C}\|f\|_{p}\left\|S^{*} g\right\|_{q} .
\end{aligned}
$$

This leads to $\|g\|_{q}=1 \leq \frac{1}{C}\left\|S^{*} g\right\|_{q}$. Thus, we can conclude that $S$ is bijective on $L^{p}(\mathbb{R}), 1<p<\infty$.

Proof of Theorem 1.2: We prove (3) (4). It is a well-known fact which every bounded unconditional basis is Besselian (Hilbertian)

$L^{p}([0,1]), 1<p \leq 2(2 \leq p<\infty)[10]$. We define

$$
I_{n}:=\left[\frac{1}{n+1} \frac{1}{n}\right), J_{n}:=[n-1, n), J_{2 n-1}:=[-n-1,-n)
$$

and there exists a linear isometric mapping $\Lambda_{n}, n \in \mathbb{N}$ frome $L^{p}\left(J_{n}\right)$ onto $L^{p}\left(I_{n}\right)$

$$
\bigcup_{n=1}^{\infty} I_{n}:=[0,1], \bigcup_{n=1}^{\infty} J_{n}:=\mathbb{R} \text {. Defing a map } \Lambda \text { from } L^{p}(\mathbb{R})
$$
onto $L^{p}([0,1])$ by

$$
\Lambda(f):=\sum_{n=1}^{\infty} \Lambda_{n}\left(f \chi_{J_{n}}\right) \text { so that } \Lambda \text { is linear, norm preserving }
$$
since

$$
\begin{aligned}
& \|\Lambda(f)\|_{L^{p}([0,1])}=\sum_{k^{\prime}=1}^{\infty}\left\|\sum_{n=1}^{\infty} \Lambda_{n}\left(f \chi_{J_{n}}\right)\right\|_{L^{p}\left(I_{k^{\prime}}\right)} \\
& =\sum_{k^{\prime}=1}^{\infty}\left\|\Lambda_{k^{\prime}}\left(f \chi_{J_{k^{\prime}}}\right)\right\|_{L^{p}\left(I_{k^{\prime}}\right)}=\sum_{k^{\prime}=1}^{\infty}\|f\|_{L^{p}\left(J_{k^{\prime}}\right)}=\|f\|_{L^{p}(\mathbb{R})},
\end{aligned}
$$

for all $f \in L^{p}(\mathbb{R})$. Therefore, $\left\{\Lambda\left(\psi_{j, k}\right)\right\}$ is also a bounded unconditional basis in $L^{p}([0,1]), 1<p<\infty$.

\section{REFERENCES}

[1] R. J. Duffin and A. C. Schaeffer, "A class of nonharmonic Fourier series," Trans. Amer. Math. Soc., vol. 72, pp. 341-366, 1952.

[2] K. Ahmad, R. Kumar, and L. Debnath, "Existence of unconditional wavelet packet bases for the spaces $L^{p}(\mathbb{R})$ and $H^{1}(\mathbb{R})$," Taiwanese J. Math., vol. 10, no. 4, pp. 851-863, 2006.

[3] E. Hernández and G. Weiss, A First Course on Wavelets, Boca Raton, Florida: CRC Press, 1996.

[4] Y. Meyer, Wavelets and Operators, Cambridge: Cambridge University Press, 1992.

[5] G. Gripenberg, "Wavelet bases in $L^{p}(\mathbb{R})$," Studia Math., vol. 106, no. 2, pp. 175-187, 1993. 
[6] Wojtaszczyk, "Wavelets as unconditional bases in $L^{p}(\mathbb{R})$," $J$. Fourier. Anal. Appl., vol. 5, no. 1, pp.73-85, 1999.

[7] N. Wiener, "Tauberian theorems," Ann. of Math., vol. 2, no. 33, pp. 1-100, 1932.

[8] O. Christensen, An Introduction to Frames and Riesz Bases, Birkhauser, Boston, 2003.

[9] E. M. Stein, Singutarlntegrals and Differentiability Properties of Functions, Princeton, NJ: Princeton University Press, 1970.

[10] I. Singer, Bases in Banach Spaces I and II, New York: Springer-Verlag, 1970.

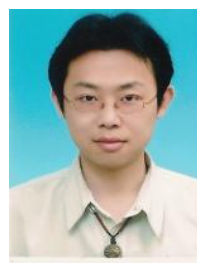

K. C. Wang received his B.S. and M.S. degrees in mathematics from National Cheng Kung University, Feng Chia University in 2000 and 2003, respectively. $\mathrm{He}$ is a researcher in Ph.D. Program in Mechanical and Aeronautical Engineering. Since 2010, he has been a lecturer in the Department of Applied Mathematics at Feng Chia University, Taiwan. His research interests include frame theory and wavelet analysis.

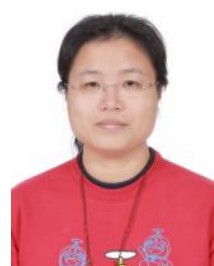

C. I. Yang received her B.S., and M.S. degrees in mathematics from National National Taichung University of Education, Feng Chia University in 1999 and 2011, respectively. Since 1999, she has been a teacher in the Dongxing elementary school, Taichung, Taiwan. Her research interests include Neural Networks and wavelet analysis.

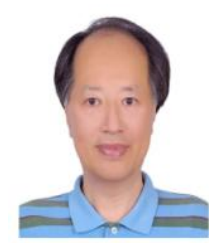

K. F. Chang received his B.S., M.S. and Ph.D. degrees in mathematics from National Taiwan Normal University, National Tsing Hua University and the University of Texas at Austin in 1979, 1981 and 1993 , respectively. Since 1998, he has been a Professor in the Department of Applied Mathematics at Feng Chia University, Taiwan. His research interests include approximation theory and wavelet analysis. 